\title{
Transposition
}

Musique et Sciences Sociales

Articles | 2020

\section{La chronique sonore d'une grève par les grévistes : décembre 1966 - février 1967 aux Avions Marcel Dassault (Bordeaux)}

The Sound Chronicle of a Strike by the Strikers: December 1966 - February 1967 at Avions Marcel Dassault (Bordeaux)

\section{Noëlle Gérôme}

\section{CpenEdition}

\section{Journals}

Édition électronique

URL : http://journals.openedition.org/transposition/5399

DOI : 10.4000/transposition.5399

ISSN : 2110-6134

Éditeur

CRAL - Centre de recherche sur les arts et le langage

Référence électronique

Noëlle Gérôme, «La chronique sonore d'une grève par les grévistes : décembre 1966 - février 1967 aux Avions Marcel Dassault (Bordeaux) », Transposition [En ligne], Articles, mis en ligne le 01 mai 2020, consulté le 02 juin 2020. URL : http://journals.openedition.org/transposition/5399 ; DOI : https:// doi.org/10.4000/transposition.5399

Ce document a été généré automatiquement le 2 juin 2020

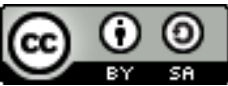

La revue Transposition est mise à disposition selon les termes de la Licence Creative Commons Attribution - Partage dans les Mêmes Conditions 4.0 International. 


\section{La chronique sonore d'une grève par les grévistes : décembre 1966 - février 1967 aux Avions Marcel Dassault (Bordeaux)}

The Sound Chronicle of a Strike by the Strikers: December 1966 - February 1967 at Avions Marcel Dassault (Bordeaux)

Noëlle Gérôme

\section{NOTE DE L'ÉDITEUR}

Le comité de rédaction de Transposition remercie le Département de l'Audiovisuel de la Bibliothèque nationale de France pour la numérisation et mise à disposition des fichiers audio qui accompagnent ce texte.

Versions antérieures de l'article :

- GÉRôME Noëlle, «Construction ouvrière d'une histoire ouvrière : culture de la grève, pratiques socio-musicologiques (décembre 1966 - février 1967 aux Avions Marcel Dassault à Bordeaux », Technologies, Idéologies, Pratiques, vol. 6, nº 2, 1986, p. 31-59. - GÉRÔME Noëlle, «Chronique sonore de l'occupation musicale d'un territoire : deux mois de grève aux usines Dassault de la région bordelaise (décembre 1966 - février 1967) », Ethnologie française, vol. 9, $\mathrm{n}^{\circ} 1$, « Musiques dans la rue : terrains de jeu », 1999, p. 49-56.

Aux mémoires de René Eyrier et d'Auguste Bouvet. 


\section{Introduction}

1 En 1982, au cours d'une campagne de recueil des productions symboliques à l'usine des Avions Dassault/ Bréguet-Aviation de Saint-Cloud, René Eyrier, délégué du personnel pour les activités culturelles au Comité d'Établissement, nous a confié un disque 33 tours hors commerce, Les fruits de la colère ${ }^{1}$, enregistré lors d'une grève qui fit date aux usines Dassault de Martignac et de Mérignac, du $1^{\text {er }}$ décembre 1966 au 28 février 1967.

2 Ce disque est consacré pour la totalité de sa première face, et pour une partie de la seconde, à l'exposé d'une chronologie de la grève par trois récitants, largement entrecoupé en illustrations par un marqueur sonore (le «tam-tam») ainsi que par des chœurs chantés et des slogans enregistrés in vivo. Il se termine, avant l'hymne de la reprise du travail, par l'enregistrement en direct de la quasi totalité du discours de Joseph Juste, représentant de la CGT à l'intersyndicale.

3 Ces chants, discours et ensembles organisés de bruits, qui composent plus de la moitié du disque, ont été enregistrés pendant l'action par certains des participants pour témoigner de l'importance de l'expression sonore et musicale dans la conduite de la grève. Objet commémoratif, édité par le Comité d'Entreprise bien après la reprise du travail, le disque n'est donc pas consacré au récit hagiographique d'une action revendicative victorieuse.

Ce média ne peut être affiché ici. Veuillez vous reporter à l'édition en ligne http:// journals.openedition.org/transposition/5399

Face A

00:00 - chronologie de la lutte : débuts

05:10 - « Tam-tam»

06:05 - suite de la chronologie

06:15 - «Si Dassault vend des Mystères » (sur l'air de "Jeanneton prend sa

faucille »)

08:48 - suite de la chronologie

09:12 - « Le travail c'est la santé, nous disent les financiers " (sur l'air de « Le

travail c'est la santé »)

12:05 - suite de la chronologie

12:35 - Hymne à la gloire des quarante heures (sur l'air de «Et on s'en fout d'attraper la vérole »)

12:55 - « Tam-tam » (seconde occurrence)

13:14 - suite de la chronologie

13:43 - «Nous irons vers la victoire» (sur l'air de «Nous ferons le tour du monde »)

15:56 - slogan « Dassault doit payer » (sur le rythme du « tam-tam »)

16:11 - suite de la chronologie

16:23 - « Oui tu l'auras ton avertissement » (sur l'air de la « Chanson de Lara »)

16:50 - suite de la chronologie

17:38 - « Si Dassault vend des Mystères » (autres couplets)

18:09 - suite de la chronologie

18:57 - « Tam-tam » (troisième occurrence), avec voix off au second plan

19:52 - suite de la chronologie

20:10 - « Si Dassault vend des Mystères » (autres couplets)

20:40 - suite de la chronologie

21:28 - « Le travail c'est la santé, nous disent les financiers » 
Ce média ne peut être affiché ici. Veuillez vous reporter à l'édition en ligne http://

Face B

00:00 suite de la chronologie (présentant ce qui suit comme des extraits de la

manifestation du 6 février 1967)

01:14 « Ohé ohé, Pompidou » (sur l'air de « Il était un petit navire »)

01:38 slogan « Dassault doit payer » (avec accélérations-décélérations)

01:57 « Des ronds, Pompom » (sur l'air de « T’as bu bonhomme »)

02:13 « Dassault si tu continues » (sur l'air de « Dites moi, les yeux languissants »)

02:29 un slogan, suivi du slogan "Dassault doit payer" (avec accélérations-

décélérations)

03:05 suite de la chronologie

04:35 récit de la perturbation par les grévistes d'un discours de J. Chaban-Delmas, entrecoupé à trois reprises par le chant «Si Dassault vend des Mystères "

07:19 fin de la chronologie

08:36 discours victorieux de Joseph Juste (CGT) en meeting

19:52 « Grâce à l'unité nos patrons abaisseront les armes » (sur l'air du « Chant des partisans »)

\section{Un témoignage sonore}

6 Aujourd'hui encore, le document demeure exceptionnel. À quelques œuvres cinématographiques près - et encore les documents sonores y sont-ils presque toujours très brefs, dominés la plupart du temps par un commentaire - ce disque constitue l'un des rares témoignages français sur les pratiques symboliques quotidiennes d'un mouvement de grève, où les chants, les slogans, l'organisation d'un univers sonore spécifique sont de coutume. Ces aspects de la vie matérielle et relationnelle, vécus dans le quotidien de la grève mais aussi dans ses temps forts et dans ses moments exceptionnels, sont généralement absents des études sur les négociations institutionnelles, bien qu'ils les aient souvent provoquées, toujours installées, " supportées » (au sens donné à ce mot dans la culture des stades), popularisées au long des semaines menant à l'aboutissement du mouvement revendicatif.

7 Tels qu'ils sont rapportés par le disque, les documents correspondent, sans médiation, au choix par les protagonistes des éléments signifiants de leur action. Autrement que l'archive de presse, et plus complètement que les archives cinématographiques, on trouve là des informations sur l'épaisseur anthropologique de l'évènement.

8 Le disque expose, au fur et à mesure de leur apparition, de leur organisation, les éléments d'un langage de résistance, expressifs au-delà des discours, véhiculant l'information et les jugements de valeurs, exprimant les compétences («certaines publications revendicatives sont des œuvres d'art, les artistes côtoient les humoristes ", dit la voix off) et, avant l'occupation de l'usine, utilisant toutes les possibilités de contourner les règles de limitation de l'expression revendicative : rythme significatif sur lequel nous reviendrons - de percussion sur du matériel de récupération dans les ateliers (le «tam-tam »), affiches, travestissements. Symbolique des bruits, de l'univers musical dont les chansons sont issues, des rythmes, des parcours, des défilés, des "accompagnements" en groupe des délégations ouvrières ou des représentants patronaux en visite, symbolique du détournement de l'usage des lieux et de la destination des cérémonies, des dons et contre-dons manifestant la solidarité... Les 
auteurs du disque et les acteurs de la grève en font le catalogue, certes incomplet, mais manifestant la volonté d'organisation, d'efficacité, d'expression symbolique, d'établissement de la mémoire d'un moment d'histoire ouvrière, et de célébration de l'éthique à laquelle se référaient projets et comportements.

9 On y entend, en situation, les différences de rythme et de ton au fil d'une allocution de fin de grève, le mélange de formalisme rhétorique et de langage direct chez le responsable syndical, la recherche des effets de communication et la nature des contacts avec la salle, les réactions de celle-ci qui souffle le slogan un instant oublié, et, comme au théâtre, le silence de son attention aux moments forts, de ses toussotements aux périodes moins tendues. Des lapsus et des répétitions dues à la fatigue, des redondances, et aussi le ton qui corrige et complète le vocabulaire, l'accent qui s'accentue ou se perd avec l'émotion, la fatigue ou la solennité de la connotation recherchée. Enfin la qualité des chœurs et des rythmes, celle des timbres de voix qui traduisent l'entraînement, le volontarisme de l'expression comme la relation à une culture locale où le groupe des hommes se célèbre habituellement par le chant, des bistrots aux férias, aux arènes ou à la pelote. Ainsi la fin des ritournelles traditionnelles des cortèges revendicatifs des années 1960 est ici ponctuée d'un "olé», issu directement de la culture des aficionados : Bordeaux est à moins de cent kilomètres de la zone d'autorisation des corridas avec mise à mort.

10 Enfin, la nature du document lui-même informe sur la maîtrise des techniques de l'enregistrement sonore, sur le raffinement de la culture technique des ouvriers de l'entreprise (le groupe le plus actif dans la grève a été celui du personnel horaire des ateliers), et sur l'étendue de l'éventail de leurs compétences, irréductibles à la qualification professionnelle manifeste, intégrant une culture musicale et sonore.

11 On le comprend, ce disque est un objet aux significations multiples, et son enveloppe, loin d'obéir aux normes de la séduction publicitaire est, de façon austère, foisonnante, didactique et organisée plastiquement, dans le prolongement de la symbolique ouvrière du $19^{\mathrm{e}}$ siècle, syncrétique et rayonnante. 


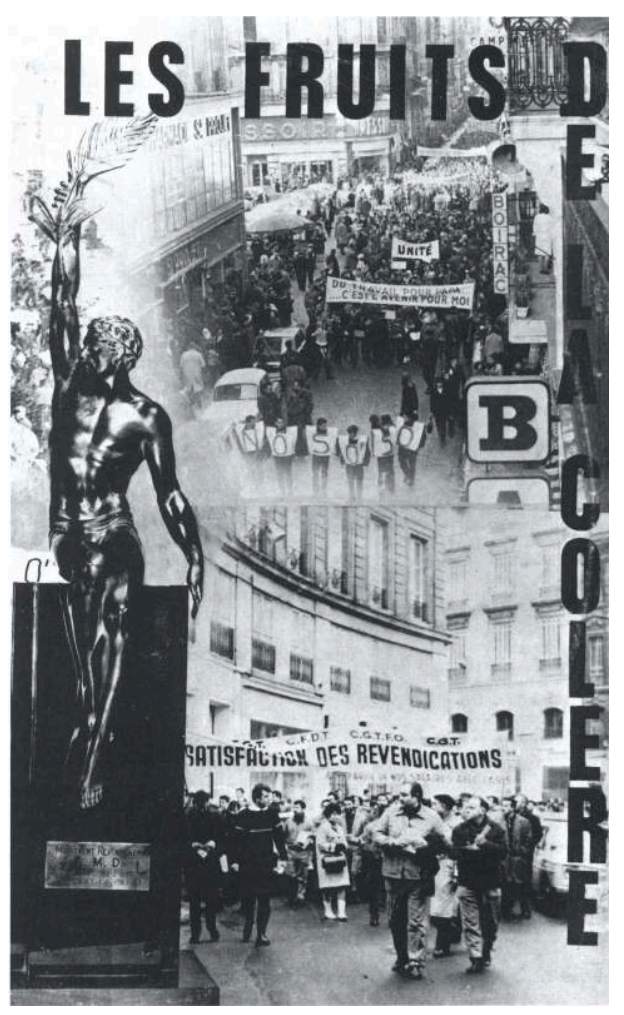

III. 1 : recto de la pochette

\section{LES FRUITS DE LA COLERE}

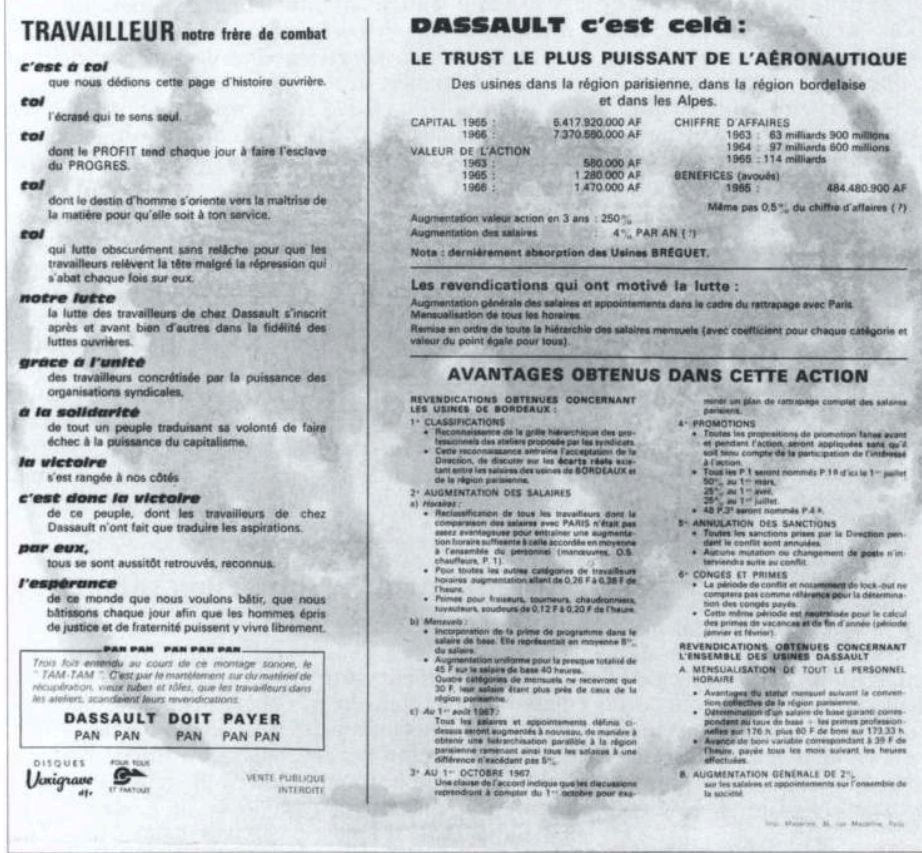

III. 2 : verso de la pochette

Les deux faces de la pochette se complètent. Au recto, quatre photographies des principales manifestations hors des usines encadrent celle de la statuette de bronze d'un génie ailé, offerte le 6 mars 1967 aux trois organisations syndicales par une jeune 
employée au nom des deux mille grévistes. Alors «la voix de Joseph Juste était émue quand il a remercié les travailleurs et les yeux se sont mouillés dans la salle, pour une formidable ovation $»^{2}$. Le verso de la pochette contient un poème qui dédie le disque "aux frères de combat" auxquels "cette page d'histoire ouvrière est adressée ". L'intention est d'inscrire l'évènement dans la généralité de la lutte des classes sociales à l'intérieur du monde capitaliste.

Un paragraphe définit rapidement le rythme, l'instrumentation et la signification du «tam-tam », martèlement sur du matériel de récupération, vieux tubes et tôles, signal sonore de la grève, que l'on entendra dans l'enregistrement à trois reprises sous cette forme, ainsi qu'en trois autres moments sous la forme criée du slogan « Das-sault doit pa-yer ».

Un autre paragraphe caractérise rapidement le Groupe Dassault en donnant l'évolution de quelques indicateurs économiques entre 1963 et 1966 : capital, valeur de l'action, chiffre d'affaires, bénéfices publiés. Les revendications à l'origine de la grève sont résumées : augmentation générale des salaires à parité avec les usines de la région parisienne, mensualisation du personnel horaire, révision de la grille hiérarchique des salaires mensuels. Toute la place est réservée aux résultats de la grève dont les revendications salariales locales sont satisfaites, où l'on revient sur les sanctions et les licenciements prévus, où les périodes de conflit et de lock-out sont indemnisées, où, enfin, la mensualisation du salaire est étendue à l'ensemble du personnel des usines Dassault, améliorant par là le calcul du salaire de base et l'augmentant de $2 \%$.

L'époque est alors à la restructuration de l'industrie aéronautique. Les sociétés nationalisées Sud-Aviation et Nord-Aviation doivent désormais composer un seul groupe : France-Aviation. On annonce, début janvier 1966, la fusion des Avions Marcel Dassault et de Bréguet-Aviation. L'action se déroule donc à Bordeaux à partir des usines Dassault, de Mérignac, de Talence, de Martignac et des Eyquems qui comptent 2200 salariés. Elle durera du $1^{\text {er }}$ décembre 1966 au 4 mars 1967, après l'échec à l'usine de Talence d'une entrevue avec le Directeur général des Avions Marcel Dassault, entraînant un débrayage suivi par l'ensemble des usines Dassault du bordelais. Le 22 décembre, les grévistes défilent dans les rues de Bordeaux. Le mouvement continue de se généraliser et s'accompagne de procédures de licenciements, mais aussi d'actions de solidarité jusqu'à l'occupation de l'usine de Mérignac le 27 janvier 1967, suivie du

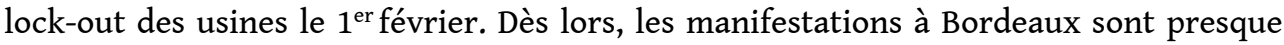
quotidiennes et obtiennent un large soutien politique, intersyndical et interprofessionnel, auquel se joint l'archevêché de Bordeaux. À l'échelle nationale, le personnel des usines Dassault est solidaire du mouvement. Le 16 février, on annonce une grève de deux heures dans la totalité de l'industrie aéronautique française. Après l'intercession de Jacques Chaban-Delmas, maire de Bordeaux, le départ du Directeur régional des usines Dassault et une hésitation de la direction sur une partie des revendications, la reprise du travail est décidée le 21 février. L'accord définitif est conclu le 29 février, avant le meeting à la Bourse du travail et le défilé de la victoire le 4 mars.

16 L'exceptionnelle longueur de la grève (trois mois), le large soutien de la société locale, celui de la totalité de l'industrie aéronautique, l'ampleur du succès des revendications, s'inscrivent dans une dynamique politique. Le temps était à la concentration des forces politiques de gauche. Au $25^{\mathrm{e}}$ congrès de la Fédération CGT des métaux le 21 novembre 1966, on note le progrès des négociations unitaires CGT-CFDT. Une journée nationale 
d'action commune sera alors envisagée pour le $1^{\text {er }}$ février 1967, à laquelle se joindra la Fédération de l'Éducation Nationale. C'est à la même période, le 21 décembre 1966, qu'est conclu l'accord entre le Parti Communiste et la Fédération de la Gauche Démocrate et Socialiste.

\section{Les éléments de la culture de la grève}

17 L'apport original et irremplaçable de ce collectage sonore et musical spontané au cœur d'une grève est de rapporter des évènements sonores dans leur contexte d'origine et de donner ainsi, sur ce qu'Alain Cottereau appelle "la culture de résistance ${ }^{3}$, des témoignages, uniques à notre sens, $d u$ domaine culturel populaire français contemporain.

\section{Les gestes et les comportements d'une culture de résistance}

Pendant les deux premiers mois de la grève, l'essentiel de l'action revendicative s'est déroulé dans les ateliers. Cette activité a choisi de se manifester par des comportements significatifs en dehors des moments formalisés des assemblées générales, des réunions syndicales et de celles des comités de lutte.

la fréquence des défilés dans l'usine même dès l'origine du conflit, qui sont un moyen de communication avec les salariés des autres secteurs de l'usine et de revendication " d'homme à homme » face à un pouvoir de décision dissimulé derrière une chaîne de médiations (direction d'établissement, direction régionale, direction nationale...).

20 L'histoire de la lutte est marquée par les défilés dans l'usine, presque autant que par les manifestations de rue. Le 27 janvier 1967, la présence du Directeur général de Dassault, conduisant une délégation étrangère, suscite un «accompagnement » tout au long du parcours dans l'usine. Au début des revendications, les 250 de Talence après « une visite des principaux bâtiments ", par un prompt renfort, se retrouvent 1000 à manifester devant le restaurant d'entreprise. Après le vote de la reprise du travail, «c'est en chantant et en cortège que les travailleurs regagnent les ateliers et les bureaux ».

21 Car il existe aussi dans les entreprises des endroits propices au départ ou à l'aboutissement des défilés, et à leur conclusion en meeting ou en manifestation: les restaurants d'entreprise ouverts à tous et regroupant le temps du repas les travailleurs des différents ateliers, le hall des bâtiments de direction dont l'appropriation de la fonction ostentatoire est un premier manquement au respect de la hiérarchie de l'usine. L'esplanade de l'île Seguin à l'usine Renault de Billancourt pourrait ainsi être considérée comme un lieu historique du mouvement ouvrier français. Sa fonction symbolique était si fortement inscrite dans les pratiques revendicatives de l'usine, qu'après la grève de 1968, la direction de l'usine a modifié le plan de l'île et fait disparaître l'esplanade.

Bien entendu, l'espace même de l'activité productive et ses espaces complémentaires (cabines, toilettes, seuils de l'usine) sont également utilisés pour des déplacements collectifs significatifs de la volonté de revendication:

- rassemblement au milieu de chaque atelier et casse-croûte en commun ;

- « arrêt-pipi, tout le monde se rendant aux toilettes à la queue leu-leu »;

- repos en commun; 
- « haies silencieuses » à la sortie du travail ou à l'entrée de la cantine sur le passage

de ceux qui n'ont pas encore rejoint le groupe des protestataires.

Il existe encore bien d'autres moyens de manifester dans les ateliers ses opinions et son désaccord : affiches revendicatives «stigmatis[ant] ici la conduite du patron - certaines sont des œuvres d'art ». Le ton employé choisit souvent le mode dérisoire : « les artistes côtoient les humoristes». Encore une façon de faire la preuve de compétences extraprofessionnelles. D'autres mises en scène qui n'emploient pas la forme du défilé, "happenings" dont les références symboliques renvoient cependant aux thèmes habituels de la culture des protestations populaires, à la fête et au deuil parodique, ici sous des formes originales: "batailles de confetti et lâchages de ballons portant des affiches revendicatives", évocations des fêtes de plein air et des carnavals urbains, " extinction des feux à huit heures » et " silence absolu ", " pause silencieuse chacun à son poste de travail », renvoyant pour leur part aux minutes de silence funèbres.

L'apparence des individus parle aussi, parfois sous forme de rébus vivants. Ce n'était pas encore le temps des autocollants et des t-shirts blasonnés, mais là «fleurissent aux boutonnières les pièces de $0,50 \mathrm{~F}$ rappelant l'augmentation demandée pour le salaire horaire ".

Ces pratiques scandent ainsi le temps de la journée dont les séquences (embauche, pause, repas, sortie...), obéissent alors à une contre-étiquette revendicative. Il se crée ainsi des formes de sociabilité qui tout à la fois expriment, diffusent et imposent des comportements, des jugements de valeur et des informations.

L'appropriation partielle de l'espace et du temps de l'usine s'exacerbera en "occupation" lors d'un nouveau refus d'entrevue après deux mois de débrayages et plusieurs réunions du Comité d'Établissement et du Comité Central d'entreprise.

\section{Porter l'information hors de l'usine}

Quand l'adversaire cherche à gagner du temps, il faut des témoins, des soutiens. Réflexe mais aussi réflexion politique. Il faut porter l'information hors de l'usine.

Les manifestations d'unité des ouvriers sortent dans la rue le 22 et le 26 décembre 1966, pour profiter dans leur publicité du climat émotionnel qui entoure les fêtes de Noël. Le mouvement s'étend le 6 février dans la région, à l'ensemble des travailleurs de la métallurgie, comprenant alors « de petites entreprises : Montbarbre, Carde, les Bennes Marrel, qui ne figuraient pas sur les registres syndicaux». Les manifestations s'élargiront encore aux familles des travailleurs le 11 février.

Le départ des délégués de l'entreprise pour des négociations à Paris ou, localement, à la mairie de Bordeaux et à l'Inspection du Travail, est toujours accompagné en cortège, pour informer le public par des manifestations spectaculaires, pour exprimer la continuité de l'unanimité, la force, la persévérance de la volonté des salariés.

Les lieux investis de l'espace public sont, comme ceux de l'usine, symboliquement significatifs, et en les occupant, les cortèges de manifestants renforcent la valeur démonstrative de leur action. De la Bourse du Travail à l'Hôtel de Ville, au Cours de l'Intendance et aux Allées de Tourny, l'itinéraire est celui de la revendication d'une légitimité, d'une visibilité.

31 Dans la ville, comme dans l'usine, on fera donc spectacle des évènements : aux dons d'argent de la population, on répond par le contre-don du sang à l'Hôpital Saint-André. 
Sur un autre mode, on envahit par surprise une inauguration municipale, dont le cours est perturbé et les participants chahutés.

Les moyens ordinaires de diffusion des revendications sont aussi utilisés : conférences de presse, comptes-rendus publics des résultats des délégations. Mais il faut souligner l'ampleur du recours à la manifestation, moyen d'expression et démonstration de l'aptitude à aménager autrement l'espace de la ville : la manifestation chante place de la République, mais elle se tait devant l'Hôpital Saint-André. On compte cinq manifestations publiques aux mois de décembre et de janvier, et en février, après le lock-out, les manifestations seront quasi quotidiennes en direction des pouvoirs publics et de l'Inspection du Travail.

Après l'usine, les ouvriers occupent la ville.

\section{La culture sonore d'une grève}

34 L'information la plus originale apportée par le disque réside dans la prise en considération et dans l'enregistrement des séquences sonores, des slogans et des chants qui expriment les revendications des grévistes sur le mode de la dénonciation parodique, de l'injonction ou de l'injure, constamment liées à l'expression musicale. La relation n'est pas fortuite. De façon à demi plaisante, les auteurs du commentaire signalent «le début des activités culturelles", termes réitérés dans le discours de reprise du travail par Joseph Juste, qui rapporte les propos des salariés appréciant les causes du succès de leur action: "d'autres disent que c'est dans la lutte contre la démoralisation qu'on a fait dans les ateliers avec toutes nos animations culturelles ». Il s'agit pour les organisateurs de «maintenir l'ambiance » dans les ateliers, de «traduire la volonté des travailleurs engagés dans l'action", d'exprimer, "dans la rue comme dans l'usine », « l'esprit des travailleurs et leur unité ».

L'organisation de l'activité musicale est révélée par l'harmonie des chœurs parlés ou chantés, la variété et la recherche des timbres des percussions ainsi que celle des rythmes. L'enregistrement du «tam-tam » présente un intérêt particulièrement rare. Nous l'avons vu, la pochette du disque le décrit comme "un martèlement sur du matériel de récupération, vieux tubes et tôles que les travailleurs dans les ateliers utilisent pour scander leur revendication: Dassault doit payer ». L'écho des ateliers contribue à la création d'un univers sonore imposant qui correspond à un autre mode d'occupation de l'espace de l'usine, opposant un rythme et des sons autrement signifiants que le bruit régulier des machines et des outils. La mention de l'emprunt des percussions du «tam-tam » aux grèves de Sud Aviation-Toulouse en 1963 témoigne d'une histoire de ces expressions sonores et de leur mémorisation. Des récits de grève, des entretiens, font quelquefois mention de ces manifestations sonores à l'intérieur de l'entreprise $e^{4}$. Mais la difficulté pour les chercheurs en sciences sociales de pénétrer dans les usines, de procéder à des observations en des moments critiques, sont des obstacles à l'enregistrement systématique de ces pratiques sonores de résistance.

Les slogans scandés obéissent au même principe d'envahissement sonore pour l'usine et pour la rue. Leur rythme varie selon les mêmes séquences que les percussions. Il s'agit d'une seule phrase qui a traversé les trois mois de l'action, dont le verbe a été modifié selon la conjoncture : " Dassault doit payer », « Dassault faut payer », « Dassault peut payer », " Dassault va payer ».Un autre slogan, qui dramatisait la situation, a été 
employé pendant le lock-out lors de la manifestation des familles: "Dassault affameur ».

Les chansons constituent l'essentiel de la culture sonore de la grève, composées sur des airs connus dans la continuité des chansons populaires. Il a été impossible de déterminer si les textes étaient les résultats de compositions collectives ou l'œuvre d'auteurs. Il faut distinguer également entre les créations propres à l'évènement et les adaptations du répertoire habituel des manifestations qui, sur l'air du «Petit navire » ou d'un vieux quadrille du Poitou, vilipendent dans les années 1960 l'économie du gouvernement Pompidou, ou promettent à Marcel Dassault, après Nixon et avec d'autres, une sortie peu honorable. Parmi les mélodies qui servent de support aux slogans des manifestations, on trouve aussi les thèmes de «T'as bu bonhomme » (« Des ronds Pompom») et de «Dites-moi les yeux languissants» («Dassault si tu continues»).

Six autres chansons accompagnent, tout au long, l'action revendicative. Les unes conjoncturelles pour protester contre une vague de sanctions au moment des premiers débrayages : «Oui, tu l'auras, ton avertissement » sur la musique de Maurice Jarre pour "La chanson de Lara » du très récent film de David Lean Le docteur Jivago (1965); les autres à tous usages comme "l'Hymne à la gloire des quarante heures", sur l'air d'une chanson de ripaille bien connue, «Et on s'en fout d'attraper la vérole ».

Trois autres chants décrivent la situation ouvrière aux usines Dassault, énumèrent les revendications et célèbrent l'action unanime des travailleurs. Pour l'un, le premier vers est construit par translation euphonique d'une chanson à la mode, « Nous ferons le tour du monde en chantant à l'unisson", qui devient là "Nous irons vers la victoire en chantant à l'unisson ». Un autre résume l'action revendicative de façon lapidaire sur un tube de l'année précédente, «Le travail c'est la santé » (1965) d'Henri Salvador :

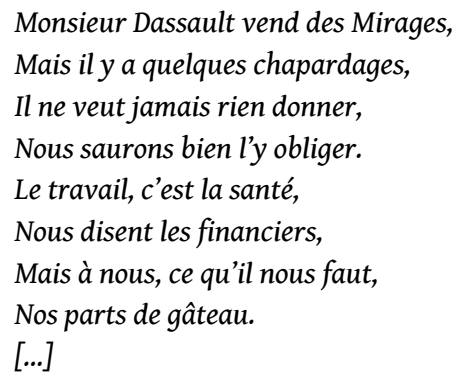

La chanson blason de la grève, celle qui a sa place dans toutes les manifestations, dans les réunions, dans les délégations, dans les ateliers, est composée sur l'air de "Jeanneton prend sa faucille», chanson au ton grivois mais qui appartient depuis des décennies au répertoire enfantin des colonies de vacances. Elle menace Benno Claude Vallières, le Directeur général des Avions Marcel Dassault, des foudres de la vindicte populaire et l'assure de la pugnacité des travailleurs de l'usine. Les couplets sont nombreux, sans liaison logique entre eux autre que la revendication, et composés, semble-t-il, au hasard des circonstances et des inspirations par des auteurs divers. Les profits de l'entreprise y sont stigmatisés, les alliances politiques du trust également, et l'on promet là aussi une juste revanche de la classe ouvrière "aux prochaines élections ».

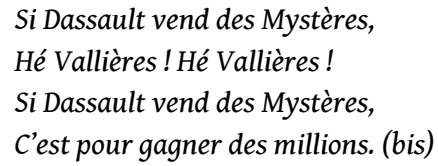




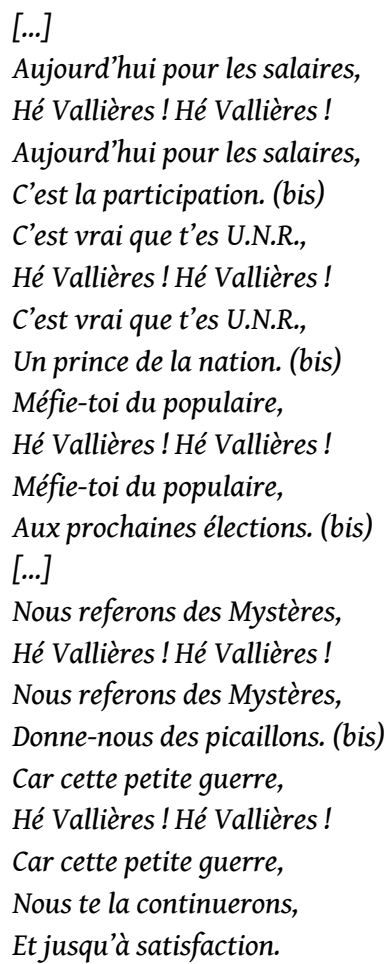

Le texte de la dernière chanson, celle composée pour le meeting de la reprise du travail, est dû vraisemblablement à un même auteur. Le texte n'a pu être transcrit qu'incomplètement car l'enregistrement est imparfait et l'ensemble des paroles peu audible. Il emprunte solennellement, pour ce grand moment, la mélodie du « Chant des Partisans ", et le chœur de voix d'hommes est à un moment accompagné d'une flûte. La partie du texte que nous avons pu transcrire exalte l'unité des travailleurs dans la lutte sociale et affirme leur invincibilité :

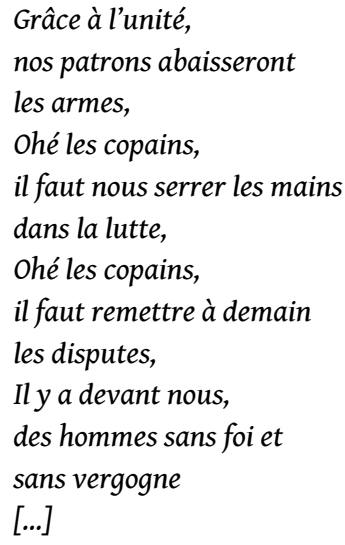

Le recours au «Chant des Partisans" renvoie à des références émotionnelles et politiques fondatrices pour le mouvement ouvrier contemporain : une longue, vaste et cruelle action de résistance victorieuse, "la Résistance ", moment de l'histoire où ce mouvement obtenait une reconnaissance et une légitimité politique, rappel également des persécutions et des sacrifices en vies humaines. L'emploi emblématique du «Chant des Partisans » n'est pas rare. Floriane Benoit, dans un livre-reportage sur les grèves de l'usine Citroën d'Aulnay d'avril à juin 1982, donne en annexe le fac-similé d'une chanson sur ce même thème - en n'utilisant qu'une partie de la mélodie -, écrite elle aussi par un O.S., à la fin de la période de grève : 


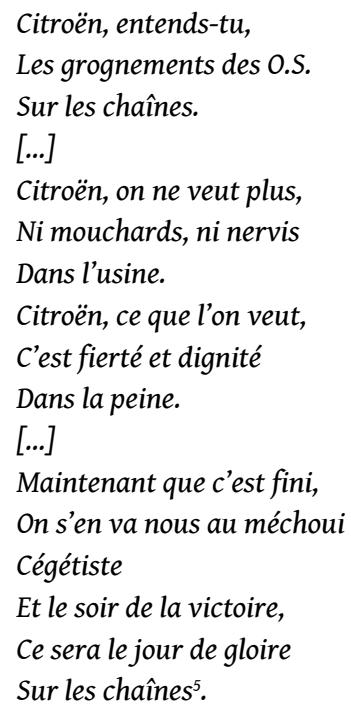

Le même esprit de célébration de la lutte des classes traverse les deux textes, chant victorieux, mais chant de bataille, atténué par l'espérance millénariste de ce que désormais, «la victoire atteinte dans l'unité, rien ne sera comme avant ». Début 2020, dans le mouvement de protestation contre le projet de réforme des retraites, c'est encore l'air du "Chant des Partisans » que l'on entendra sur les marches de l'Opéra Bastille. Le disque s'achève sur ce chant, avec un effet sonore d'éloignement mis en place à l'enregistrement.

On s'étonnera dans ces évocations multiples de l'histoire du mouvement ouvrier de n'entendre à aucun moment «l'Internationale ». La dynamique intersyndicale de la grève en est sans doute l'explication. Les allusions à l'activité mobilisatrice de la C.G.T. sont transparentes, pourtant on ne cite pas précisément les forces politiques engagées aux côtés des grévistes, on désigne sans préciser des " partis politiques de gauche » et des «formations démocratiques". Dans une volonté d'unanimité très forte, la référence à «l'Internationale » pouvait apparaître à la fois comme trop idéologique, abstraite, trop précisément connotée. Si la lettre des discours, les textes des chansons et des slogans ne sont aucunement contradictoires avec la ligne de la C.G.T. et du Parti Communiste, l'expression est plutôt celle d'un climat émotionnel unitaire relatif à des évènements concrets, précisément identifiables, d'une grève réussie à Sud-Aviation à la Résistance...

\section{Conclusion}

Si limitée que soit l'étude descriptive du contenu de ce disque, elle renvoie à deux problématiques encore mal explorées : celle de l'univers de la culture ouvrière, et celle de l'histoire originale d'un mouvement revendicatif, de l'agencement symbolique du langage et des relations de sociabilité, des déplacements, des bruits de la vie quotidienne, de l'environnement et du capital musical des ouvriers d'une usine. Ainsi dans ces circonstances particulières se constitue l'œuvre culturelle d'un groupe, même si tous les éléments n'en sont pas originaux mais insérés dans une pratique sociopolitique spécifique. Au demeurant, quelle culture de quel groupe est-elle originale? L'étude du mouvement ouvrier est aussi celle de ce creuset, pour employer une métaphore usée mais efficace, où sont appropriés, transcrits et intégrés à d'autres 
données, des résultats de spéculations intellectuelles originales, ainsi que des informations parvenues au groupe, des observations et des études qui lui sont propres. $\mathrm{Au}$ groupe, ou aux groupes? Il semble bien que l'on se trouve devant une pluralité de phénomènes dont il faudrait pouvoir organiser l'étude.

Il faudrait encore pouvoir affiner la connaissance de l'histoire de la culture musicale de cette grève dont on a pu voir qu'elle s'inscrivait dans l'ensemble culturel revendicatif d'une industrie («tam-tam » venu des grèves de 1963 de Sud-Aviation Toulouse), luimême inclus dans une culture revendicative plus large (transformation du "Chant des Partisans ", « scies » des manifestations), comme dans une pratique musicale populaire empruntant soit aux produits de l'industrie culturelle, soit aux mélodies véhiculées par le système scolaire, soit encore à celles des souvenirs familiaux ou des sociabilités locales.

Il semble également que la pleine signification de la culture de l'évènement, tel qu'on le trouve rapporté dans des chroniques comme celle que constitue ce disque, dans des archives de presse ou de police, ne puisse être établie sans la connaissance de l'histoire quotidienne de cet événement, et sans la connaissance de l'histoire quotidienne du groupe qui le vit.

Qu'est-il advenu ensuite? Pendant combien de mois encore et dans quelles circonstances a-t-on continué de fredonner certains de ces airs, de frapper certains rythmes? À l'occasion de quels rassemblements ont-ils jailli ou ont-ils été imités ? Quels en étaient les auteurs ou les groupes d'auteurs? Y a-t-il eu des suites à ce foisonnement de créations? Inversement, comment en quatre ans le «tam-tam » avaitil été transporté de Toulouse à Bordeaux ? Y avait-il, avec une moindre publicité et pour une plus petite audience, dans les années qui ont précédé la grève, des auteurs, ou des auteurs-compositeurs qui trouvaient dans l'usine une source d'inspiration et un public ? Une telle éventualité ne serait pas rare. Il existe des auteurs-compositeurs dans les entreprises dont les talents sont connus et sollicités. Mais existait-il aussi des jeux collectifs de création langagière ou musicale dans certains groupes de travail ? Dans ces sociétés d'hommes où l'expression musicale et dansée est habituelle, la transposition d'une pratique sociale à une pratique ouvrière revendicative est vraisemblable.

Ainsi des informations, des représentations seraient-elles confrontées aux circonstances de la vie quotidienne dans l'usine, soumises aux déterminations des circonstances socio-économiques et à celles du formalisme social accepté et secrété par le groupe, traduites enfin et peut-être amplifiées sur des modes expressifs immédiatement diffusables et accessibles à tous les travailleurs (enregistrement sonore, cinématographique, vidéographique, photographique, expression plastique...). C'est dans l'étude de ce mouvement d'établissement et de renforcement de systèmes d'expressions que réside, selon nous, le domaine de l'anthropologie de la culture ouvrière.

\section{Remarques d'un autre temps. Post-face}

Redoutable exercice que d'avoir à republier un article écrit il y a plus de trente-cinq ans, à propos d'évènements qui se sont déroulés il y a plus d'un demi-siècle. Ainsi que je l'ai écrit, le document sonore d'enregistrement des sons d'une grève par les ouvriers eux-mêmes, m'avait été remis par René Eyrier, élu au Comité Central d'Entreprise des usines Dassault, dont je ne célébrerai jamais assez la générosité et la clairvoyance. À 
l'époque de la rédaction de l'étude, en poste au Centre d'Ethnologie Française du Musée National des Arts et Traditions Populaires, je mettais en place un programme d'étude des productions symboliques ouvrières.

Depuis le moment de l'enregistrement, les usines Marcel Dassault ont traversé les bouleversements du tissu industriel français, la population des salariés de l'industrie métallurgique et leur culture propre ont été fondamentalement transformées. L'importance et l'influence des organisations syndicales, leur mode d'action dans leurs relations avec le patronat, ont radicalement évolué. Hors des procédures de négociations, il semble maintenant, à l'observation, que les épreuves de force passent par les manifestations dans les espaces extérieurs aux lieux de travail, avec le recours à la diffusion audiovisuelle de l'évènement la plus large possible. Ici, le « son » de la grève s'insère dans les bruits de la ville et les domine. Il accompagne les « performances » des grévistes, autres instruments de visibilité.

Malgré les modifications des formes de la "contestation", sur le temps long, on remarquera la permanence de références musicales et sonore de résistance. Avec «le Chant des Partisans ", le "Chant des marais » (Moorsoldatenlied) créé en 1933 en Allemagne dans un camp d'internement politique, repris en 1939 par les Brigades Internationales de la Guerre d'Espagne, appartenant ensuite aux chants des camps d'extermination, a été adapté dans les années 1970 par les mouvements féministes, et est repris en 2020 comme "Chant des femmes ». Depuis les années 1980, "Bella Ciao », le chant protestataire des mondine (ouvrières saisonnières) des rizières du Pô, sous sa forme adaptée par les partisans italiens, remplace "l'Internationale». Et, dans un registre chanté plus bref, le slogan contre la Réforme Devaquet en 1985 : « Devaquet, si tu savais/ ta réforme, ta réforme/ Devaquet, si tu savais/ ta réforme où on s'la met/ $\mathrm{Au}$ cul, au cul, aucune hésitation! », est encore utilisé en 2020, trente-cinq ans plus tard.

Les fruits de la colère sont un document unique que l'on ne retrouvera dans aucune étude globale des sons «sur le vif» d'une revendication salariale, sons innovateurs et réinterprétant une mémoire venue de loin, occultée et quelquefois interdite.

\section{BIBLIOGRAPHIE}

BERCE Yves-Marie, Croquants et nu-pieds : les soulèvements paysans en France du XVI $I^{e}$ au XIX $X^{e}$ siècle, Paris, Gallimard, 1974.

BERMANI Cesare, Bella Ciao. Storia e fortuna di una canzione: dalla resistenze italiana all'universalità delle resistenze, Novara, Interlinea, 2020.

BONNET Serge, La ligne rouge des hauts-fourneaux : grèves dans le fer lorrain en 1905, Paris, DenoëlSerpenoise, 1981.

BRÉCY Robert, Florilège de la chanson révolutionnaire de 1789 au Front Populaire, Paris, Hier et Demain, 1972.

CALVET Louis-Jean, La production révolutionnaire : slogans, affiches, chansons, Paris, Payot, 1976. 
CAZALs Rémy, Avec les ouvriers de Mazamet dans la grève et dans l'action quotidienne, 1909-1914, Paris, Centre d'Histoire du Syndicalisme / Maspero, 1978.

COLLET Serge, « La manifestation de rue comme production culturelle militante », Ethnologie française, vol. 12, $\mathrm{n}^{\circ} 2,1982$, p. 167-176.

COTTEREAU Alain, préface à la réédition de POULOT Denis, Le Sublime ou le travailleur comme il est en 1870, et ce qu'il peut être, Paris, Maspero, 1980 [1870].

GÉRÔME Noëlle, « Rituels contemporains des travailleurs de l'aéronautique », Ethnologie française, vol. 14, n 2, 1984, p. 177-196.

GÉRÔME Noëlle, « Elle est à toi cette chanson », Spécial options, n 7, 1984.

PERROT Michelle, Les ouvriers en grève : France 1871-1890, Paris-La Haye, Mouton, 1974.

PIGENET Michel, « Espace et rituel de la protestation dans les campagnes au XIX siècle : l'exemple du Cher », Révoltes et Société, t. 1, Paris, Les Publications de la Sorbonne, 1989, p. 283-290.

QUIÈVRE Adrien, « Les espaces politiques du paysage sonore : écouter les grèves minières au XIX siècle ", MEHL Véronique et PÉAUD Laura (dir.), Paysages sensoriels : approches pluridisciplinaires, Rennes, PUR, 2019, p. 87-98.

RIVIÈRE Henri-Georges, « Histoire de la chanson populaire : entretien avec Jean Dréjac », Almanach de l'Humanité, 1976.

TARTAKOWSKY Danielle, Le pouvoir est dans la rue : crises politiques et manifestations en France, Paris, Aubier, 1998.

TARTAKOWSKY Danielle, Les manifestations de rue en France : 1918-1968, Paris, Les Publications de la Sorbonne, 1998.

TILLY Charles, La France conteste, de 1600 à nos jours, Paris, Fayard, 1986.

VOVELLE Michel, La mentalité révolutionnaire : société et mentalités sous la Révolution française, Paris, Messidor, 1985.

\section{NOTES}

1. Les fruits de la colère, s. 1., s. d. (Voxigrave V6937).

2. L'Humanité, 7 mars 1967.

3. COTTEREAU Alain, préface à la réédition de POULOT Denis, Le Sublime ou le travailleur comme il est en 1870, et ce qu'il peut être, Paris, Maspero, 1980 [1870].

4. Il a notamment existé un instrument utilisant les propriétés sonores du titane : «le Scorpion de Saint-Nazaire » (collecte Noëlle Gérôme, enquête productions symboliques ouvrières, Avions Marcel Dassault-Bréguet Aviation, 1983). En 1979, au colloque d'ethnomusicologie de l'Institut culturel italien à Paris, une communication de l'Institut Ernesto di Martino portait sur le langage des percussions de l'usine d'armement de la F.L.O.G. à Florence.

5. BENOIT Floriane, Citroën, le printemps de la dignité, Paris, Éditions Sociales, 1982. 


\section{RÉSUMÉS}

Lors d'une longue grève aux usines Dassault-Aviation de Martignac et de Mérignac ( $\mathrm{du} 1^{\mathrm{er}}$ décembre 1966 au 28 février 1967), des ouvriers de l'intersyndicale ont enregistré leurs chants et l'un des marqueurs sonores des revendications, le «tam-tam» qui résonne dans les ateliers au rythme martelé de "Das-sault doit pa-yer». La conservation de ces rares témoignages qui attestent de l'importance de l'expression sonore et musicale dans la conduite de la grève a été assurée par l'auto-édition d'un disque 33 tours commémoratif, Les fruits de la colère, où les enregistrements in vivo sont intégrés à un récit chronologique de la lutte. Noëlle Gérôme, ethnologue du domaine français au CNRS, transcrivait et analysait en 1986 l'intégralité du document pour la revue Technologies, Idéologies, Pratiques, et en valorisait un extrait en 1999 dans les actes d'un colloque de la Société d'Ethnologie Française. Elle y présentait l'importance sociopolitique des productions symboliques ouvrières, la richesse des cultures sonores au sein des usines et les compétences de leur personnel dès lors qu'il s'agit, au delà de leurs fonctions professionnelles, de produire et enregistrer leur univers sonore. Révisée par l'auteur sous une forme inédite, cette republication en ligne offre au lecteur l'accès à l'intégralité du document audio.

During a long strike at the Dassault-Aviation factories in Martignac and Merignac (from 1 December 1966 to 28 February 1967), workers from the inter-union coalition recorded their songs and one of the soundmarks of the demands, the "tam-tam" which echoed in the workshops to the hammered rhythm of "Das-sault doit pa-yer". The preservation of these rare testimonies, which attest to the importance of sound and musical expression during the strike action, was ensured by the self-release of a commemorative 33-rpm vinyl, Les fruits de la colere, where the in vivo recordings are integrated into a chronological account of the struggle. Noëlle Gérôme, an ethnologist in the French field at the CNRS, transcribed and analysed the entire document in 1986 for the journal Technologies, Idéologies, Pratiques, and in 1999 she used an extract from it in the proceedings of a symposium of the Société d'Ethnologie Française. She presented the sociopolitical significance of symbolic worker productions, the richness of sound cultures within factories and the skills of their personnel when it comes to producing and recording their sound universe beyond their professional functions. Revised by the author in an unpublished form, this online republication offers the reader access to the entire audio document.

\section{INDEX}

Mots-clés : grève, culture sonore, chanson, manifestation, culture ouvrière, Dassault Aviation Keywords : strike, sound culture, song, demonstration, working-class culture, Dassault Aviation

\section{AUTEUR}

\section{NOËLLE GÉRÔME}

Noëlle Gérôme est ethnologue du domaine français, d'abord au Centre d'Ethnologie Française du Musée National des Arts et Traditions Populaires puis au Centre d'Histoire des Mouvements Sociaux et du Syndicalisme (CHS, CNRS / Université Paris 1 Panthéon-Sorbonne). Avec les encouragements de Georges Henri-Rivière, fondateur du MNATP, elle a mis en place un programme d'étude des productions symboliques ouvrières, qui s'appuie depuis 1979 sur un 
séminaire mensuel et a donné lieu à un bulletin annuel entre 1995 et 2005 : La Région Parisienne, industrielle et ouvrière (DRAC Île-de-France / RATP). Ce séminaire se prolonge aujourd'hui, témoignant de la fécondité de la perspective de recherche, sous la direction de Sylvie Zaidman (directrice du Musée de la Libération de Paris Leclerc-Moulin) et d'Emmanuel Bellanger (directeur du CHS), avec le soutien de la RATP, des Archives de France, des Archives de Paris, des Archives de la Seine-Saint-Denis et du CHS. Noëlle Gérôme a par ailleurs publié, avec Danielle Tartakowsky, La Fête de l'Humanité, culture communiste, culture populaire (Messidor-Éditions Sociales, 1988) ; et avec D. Tartakowsky et Alain Corbin, Les usages politiques des fêtes aux XIX $\mathrm{XX}^{e}$ siècles (Publications de la Sorbonne, 1994). 